\title{
The Prevalence of Synthetic Color in Saffron and SweetProducts (Yellow Halvah, Candy and Crystallized Sugar) Supplied in Store of Hamadan City Using Thin Layer Chromatography (TLC)
}

\section{Mohamad Moghadasi (MSc) ${ }^{1}$, Alireza Rahimi (Msc) ${ }^{1}$, Ali Heshmati (PhD) ${ }^{2, *}$}

${ }^{1}$ MSc in Food Safety and Hygiene, Student Research Center, Hamadan University of Medical Sciences and Health Services, Hamadan, Iran

${ }^{2}$ Associate Professor of Departmet of Nutrition and Food Safety, School of Medicine, Nutrition Health Research Center, Hamadan University of Medical Sciences and Health Services, Hamadan, Iran

* Corresponding Author: Ali Heshmati, Departmet of Nutrition and Food Safety, School of Medicine, Nutrition Health Research Center, Hamadan University of Medical Sciences and Health Services, Hamadan, Iran.Email: a.heshmati@umsha.ac.ir

\begin{tabular}{|c|c|}
\hline & Abstract \\
\hline $\begin{array}{l}\text { Received: } 13 / 08 / 2018 \\
\text { Accepted: } 15 / 12 / 2018\end{array}$ & \multirow[b]{2}{*}{$\begin{array}{l}\text { Background and Objective: In recent years, the use of synthetic color has } \\
\text { considerably grown instead of saffron in food products. This problem can } \\
\text { be a serious concern for consumer health. This study was conducted to } \\
\text { determine the presence and type of synthetic color in saffron based or } \\
\text { flavored products. } \\
\text { Materials and Methods: This cross-sectional study was carried } \\
\text { out on } 96 \text { samples including saffron, yellow halvah, candy, and } \\
\text { crystallized sugar (each } 24 \text { samples) that were randomly sampled } \\
\text { from stores in Hamadan city. The qualitative determination of the } \\
\text { colors including quinoline, sunset yellow, tartarazine, azorubine, } \\
\text { brilliant blue, ponceau } 4 \mathrm{R} \text { and allura red was done by thin layer } \\
\text { chromatography (TLC). } \\
\text { Results: Out of } 96 \text { total samples, } 64 \text { (66.67\%) samples had synthetic color } \\
\text { and } 32 \text { (33.33\%) samples had natural color. Nineteen (79.16\%), } 20 \\
\text { (83.33\%), } 19 \text { ( } 79.16 \% \text { ) and } 6 \text { ( } 25 \% \text { ) of saffron, yellow halvah, candy, and } \\
\text { crystallized sugar samples, respectively, contained artificial color. Among } \\
\text { used synthetic colors, the quinoline was the most abundant used color. } \\
\text { Conclusion: Based on the results of this study, } 66.67 \% \text { of the samples had } \\
\text { synthetic color, which indicates the utilization of artificial color in the } \\
\text { studied products is high. Therefore, it is recommended to control highly } \\
\text { these products. }\end{array}$} \\
\hline $\begin{array}{l}\text { How to Cite this Article: } \\
\text { Moghadasi M, Rahimi A, } \\
\text { Heshmati A. The Prevalence of } \\
\text { Synthetic Color in Saffron and } \\
\text { SweetProducts (Yellow Halvah, } \\
\text { Candy and Crystallized Sugar) } \\
\text { Supplied in Store of Hamadan } \\
\text { City Using Thin Layer Chroma- } \\
\text { tography (TLC). Pajouhan } \\
\text { Scientific Journal. 2019; 17(2): } \\
\begin{array}{l}\text { 31-36. DOI: 10.29252/psj. } \\
\text { 17.2.31 }\end{array}\end{array}$ & \\
\hline & $\begin{array}{l}\text { Keywords: Candy; Crystallized Sugar; Hamadan; Saffron; Synthetic Color; } \\
\text { Yellow Halvah }\end{array}$ \\
\hline
\end{tabular}


dof: $10.29252 /$ psj.17.2.31

\section{بررسى وجود و نوع رنكهاى مصنوعى در محصولات غذايى بر هايه يا طعم داده شده با}

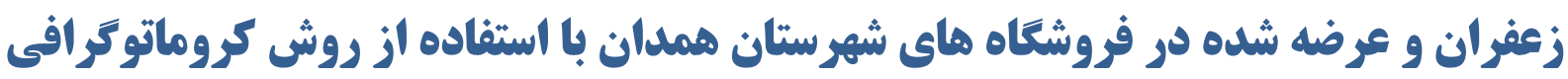

محمد مقدسى'! عليرضا رحيمى' ؛ على حشمتى ז.**

' كارشناسى ارشد بهداشت و ايمنى مواد غذايى، مركز تحقيقات دانشجويان، دانشكاه علوم يزشكى همدان، همدان، ايران

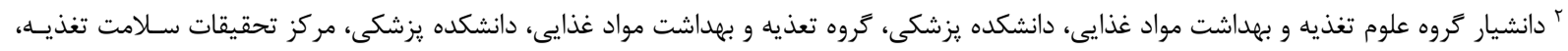
دانشخاه علوم يزشكى همدان، همدان، ايران

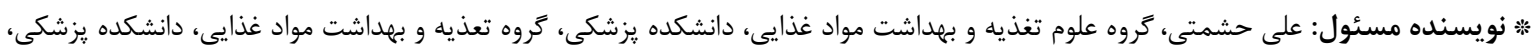

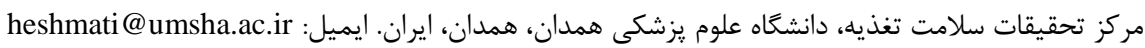

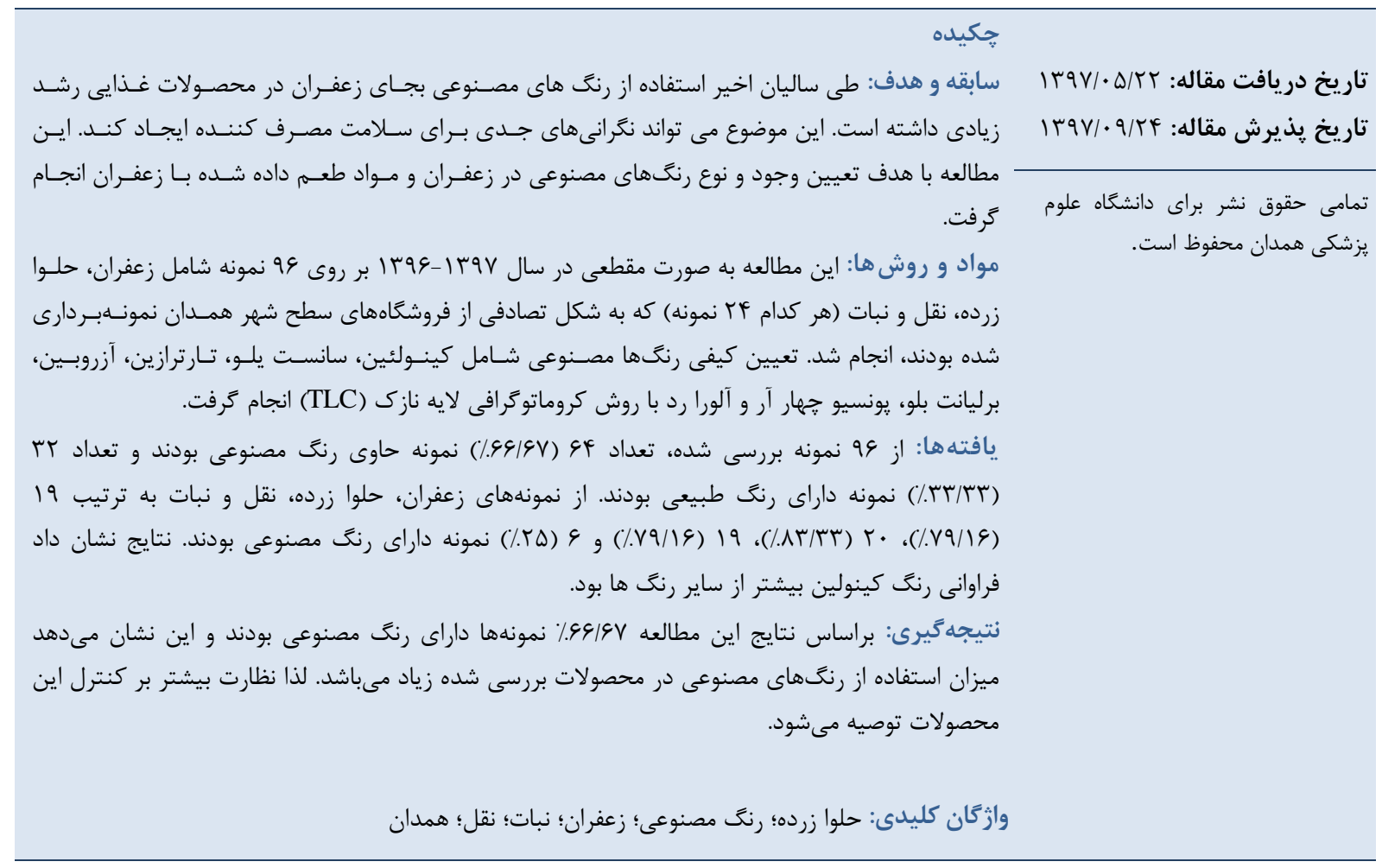

مقلدمه

C و ساير تركيبات مفيد مىباشد، مسىتوانـد نقـش بسـزايى در

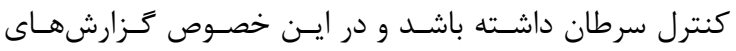

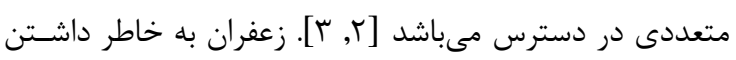

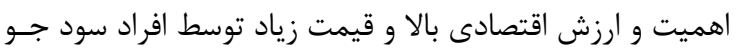

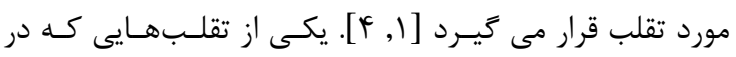

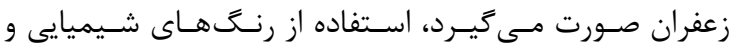

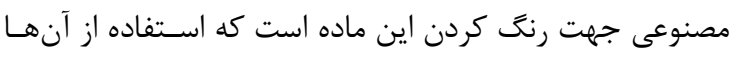

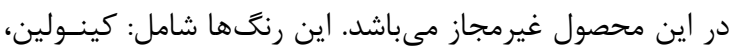

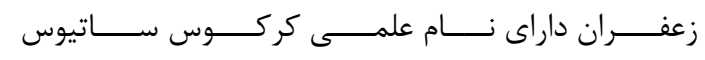

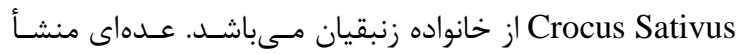
زعفران را ايران مى دانند، برخى از محققين نيز خاستعاه زعفران

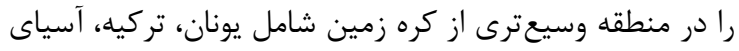

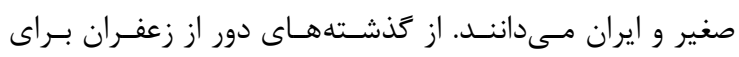
افزايش رنت و طعم مواد غذايى استفاده مىشود [1 إن. استفاده از

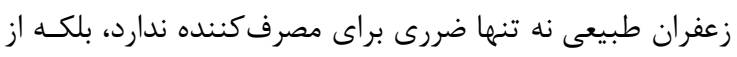

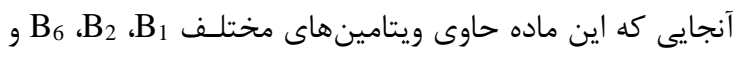




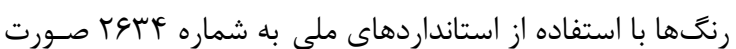

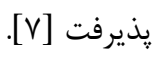

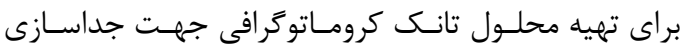
رنتها، • 1 حجم بوتانول نرمال + ه حجـهم اسـيد اسـتيك +

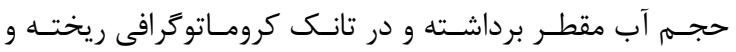

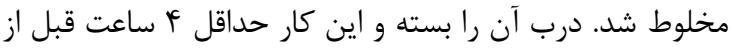

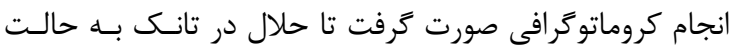

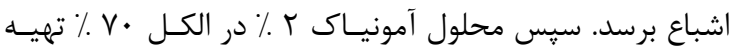
شد [V] ش

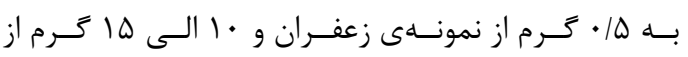

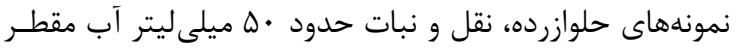

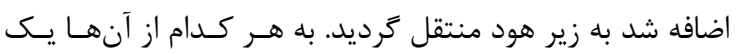

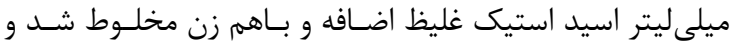

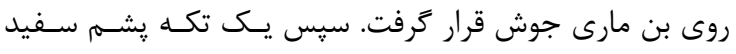

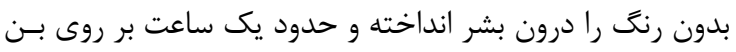

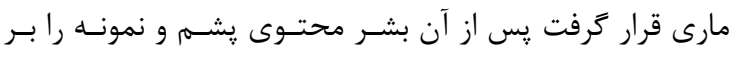

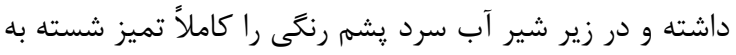

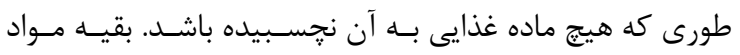

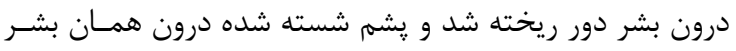

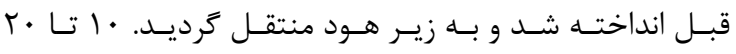

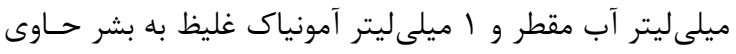

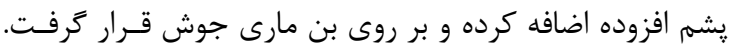

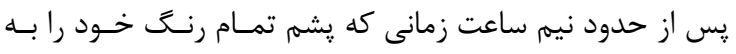

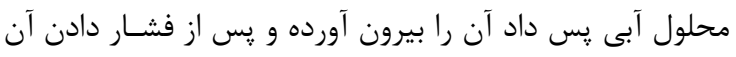

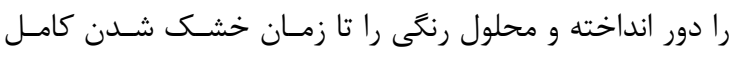
روى بن مارى باقى ماند [V]

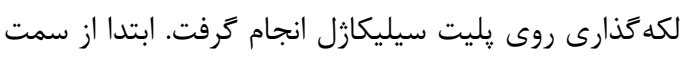

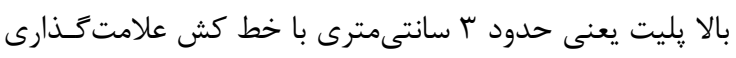

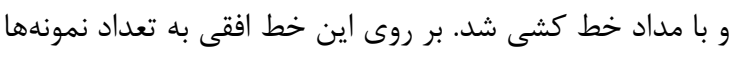

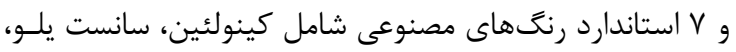

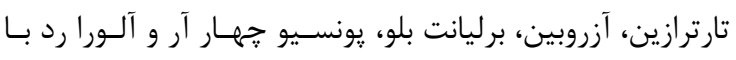

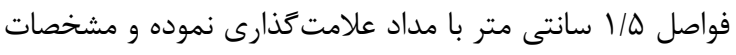

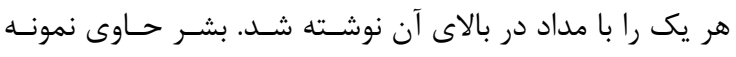

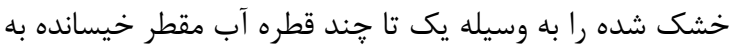
نحوى كه رنت خشك شده ته ظرف در آن حل شود و به وسيله وسيله

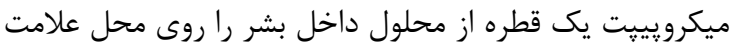

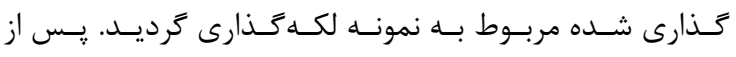

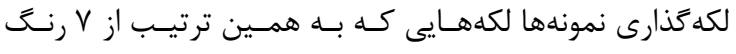
استاندارد در بوتانول نرمال تهيه شده بود بر روى رئه نقاط مشخص

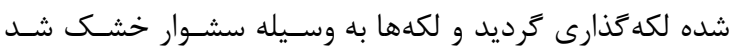

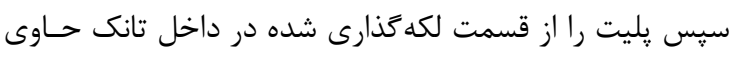

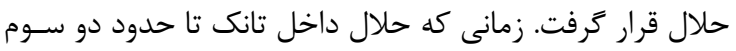

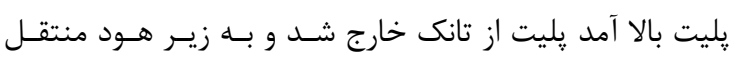

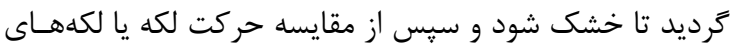

تارترازين، آمارانت، سانست يلو و يونسيو مىباشد [ه]. رنغ غذا نقش زيادى در جلب توجه مصرف كننده دارد؛ بنابراين اسـتفاده إناه

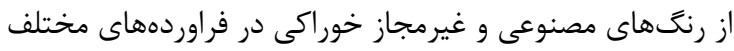

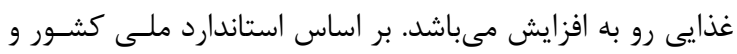

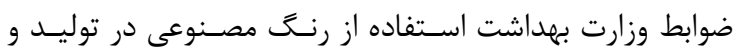
فراورى اغلب محصولات غذايى بجز موارد محدود نظير نوشابه و

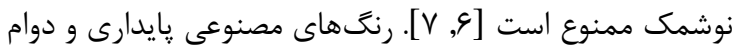

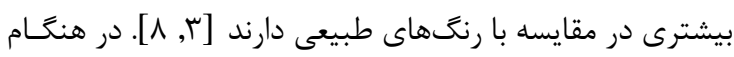

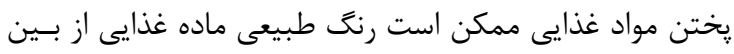

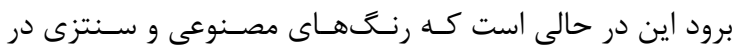

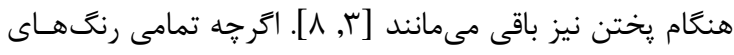

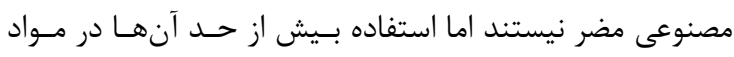
غذايى و يا ساير فراوردهها مى توانـد مشـكلات متعـددى ايجــاد نمايد. رنگهاى مصنوعى در محصولات غذايى مسىتوانسـ اثـرات

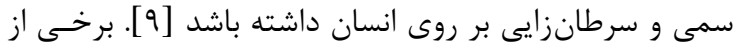

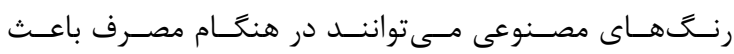

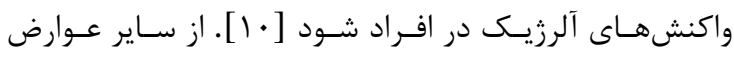

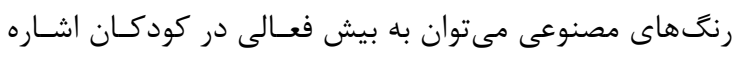

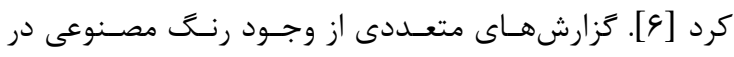

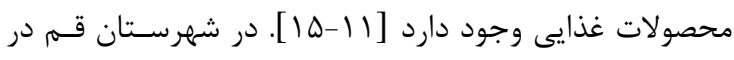

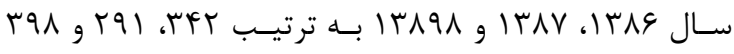
نمونه ار محصولات مختلف عرضه شده در قنادى نمونه بردارى

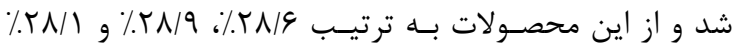

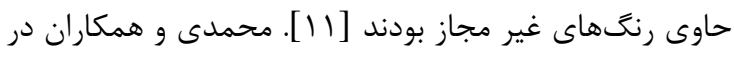

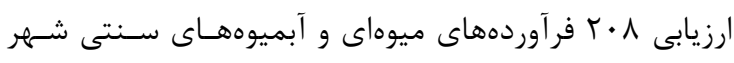

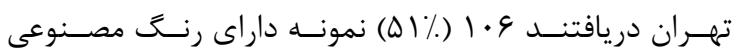

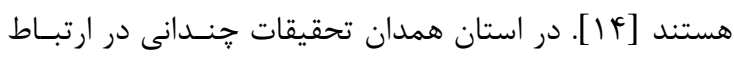

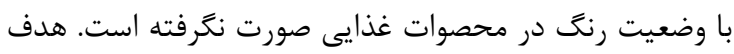

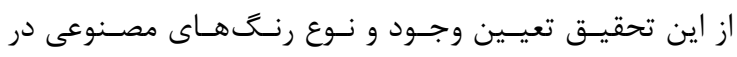

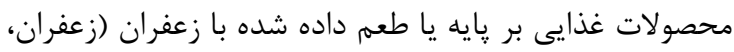

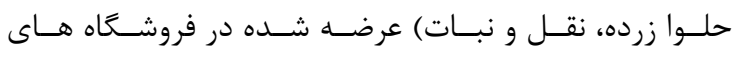

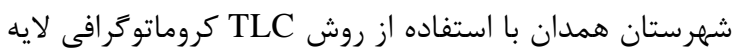

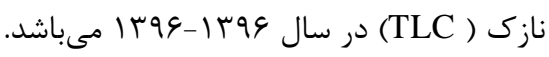

\section{مواد و روشها}

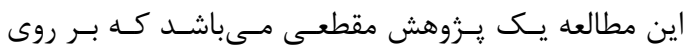

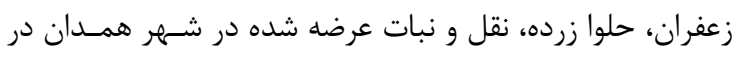

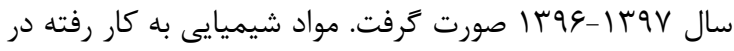

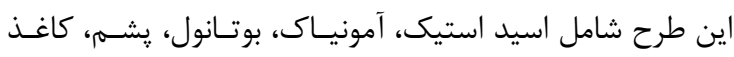

كروماتوكرافى بود كه همكى از شركت مركى آلمان تهييه شد.

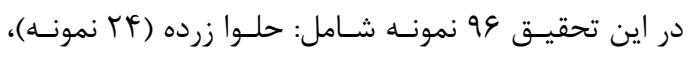

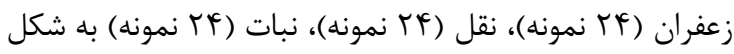

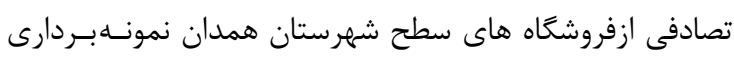

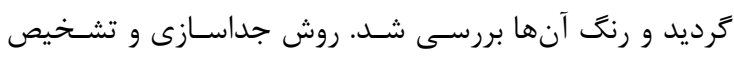


در آزمايشهاى صورت كرفته بر روى زعفـران، حلـوا زرده،

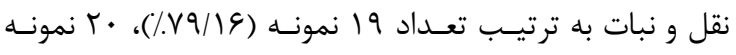

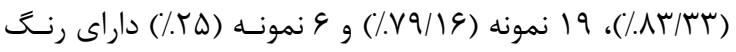

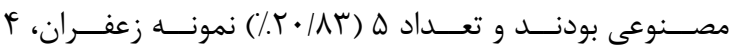

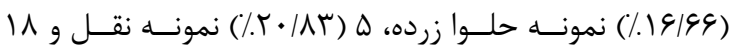

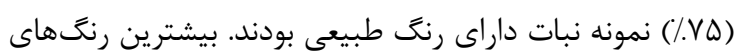

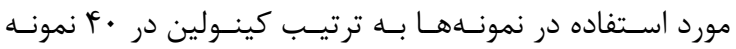

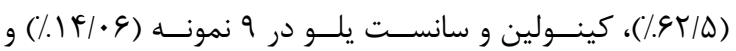

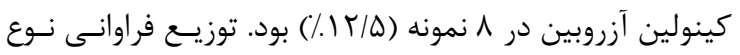

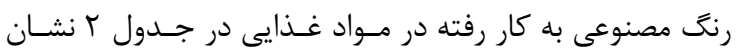
داده شده است.
نمونه با لكههاى رنخَهاى استاندارد نـوع رنـــهـاى مصـنوعى

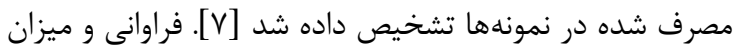

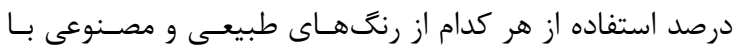

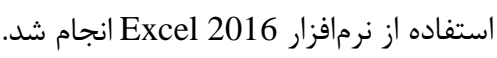

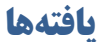

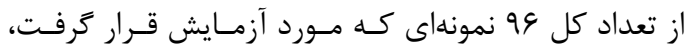

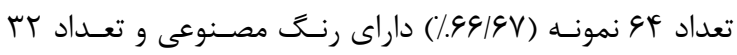

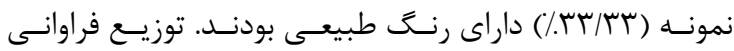
نمونهها بر حسب وجود رنگ مصـنوعى و طبيعسى در جـدول 1 بيان شده است.

جدول (: توزيع فراوانى نمونه ها بر حسب نوع رنَ (طبيعى، مصنوعى)

\begin{tabular}{|c|c|c|c|}
\hline تعداد نمونه دار اى رنَ مصنوعى (\%) & تعداد نمونه داراى رنَ طبيعى (\%) & تعدادكل نمونه آناليز شده & 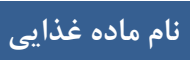 \\
\hline $19(/ . V / 19)$ & $\Delta(/ / r \cdot / \Lambda r)$ & TF & زعفران \\
\hline$r \cdot(/ . \Lambda \Gamma / \Gamma r)$ & $f(/ .19199)$ & TF & حلوازرده \\
\hline $19(/ . \vee 9 / 19)$ & $\Delta(/ . T \cdot / \Lambda r)$ & rF & نقل \\
\hline$G(/ . r \Delta)$ & $1 \wedge(/ \vee \vee \Delta)$ & TF & نبات \\
\hline $94(1.99 \mid 9 \mathrm{~V})$ & (זس/ & 99 & مجموع \\
\hline
\end{tabular}

جدول r: توزيع فراوانى نوع رنگ مصنوعى به كار رفته در انواع محصولات غذايى

\begin{tabular}{|c|c|c|c|c|c|}
\hline \multirow[t]{2}{*}{ 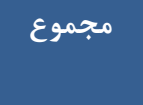 } & \multicolumn{4}{|c|}{ نوع ماده غذايى } & \multirow{2}{*}{ نوعداد نمونه دار ايى رنكَ مصنوعى و } \\
\hline & 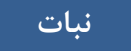 & 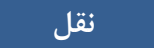 & حلوازرده & زعفران & \\
\hline \multirow[t]{2}{*}{ gq } & 4 & 19 & $r \cdot$ & 19 & تعداد نمونه داراى رنگ مصنوعى \\
\hline & & & & & نوع رنت مصنوعى \\
\hline$F \cdot(/ . g Y / \Delta)$ & $9(/ .1 \cdots)$ & $q(/ / F V / T \varphi)$ & $19(/ . \Lambda \cdot)$ & $q(/ . F V / \mu \varphi)$ & كينولين \\
\hline • & - & - & - & - & سانست يلو \\
\hline · & - & - & - & - & 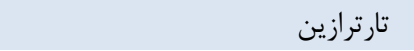 \\
\hline $1(/ 1 / \Delta 4)$ & - & $1(/ . \Delta / T \varphi)$ & - & - & 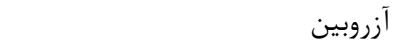 \\
\hline $9(/ .14 / .9)$ & - & $r(/ / 1 \Delta / \Delta V)$ & - & $9(/ . Y N / \Delta V)$ & كينولين و سانست يلو \\
\hline$\wedge(/). Y / \Delta)$ & - & $r(/ .1 \cdot / \Delta r)$ & $F(/ / r \cdot)$ & $r(\%) \cdot / \Delta T)$ & كينولين و آزروبين \\
\hline$r(/ / F / 9 \Lambda)$ & - & $r(/ .1 \cdot \mid \Delta r)$ & - & $1(/ . \Delta / Y \varphi)$ & تارترازين و سانست يلو \\
\hline $1(\% / 109)$ & - & $1(/ .1 / \Delta 9)$ & - & - & سانست يلو و برليانت \\
\hline $1(/ .1 / \Delta 9)$ & - & $1(/ .1 / 09)$ & - & - & آزروبين، سانست يلو و برليانت \\
\hline $1(/ .1 / \Delta 9)$ & - & - & - & $1(/ .1 / \Delta 9)$ & تارترازين و آزروبين \\
\hline
\end{tabular}

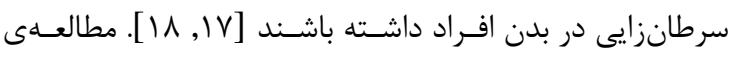

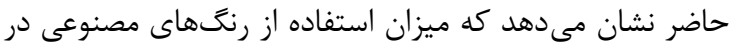

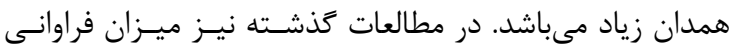

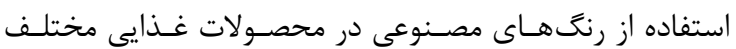

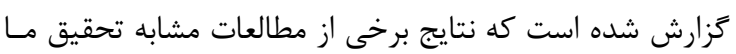

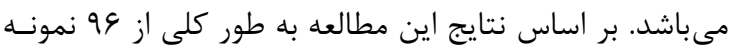

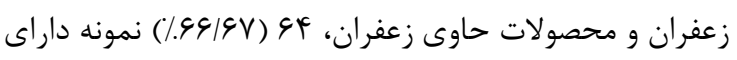

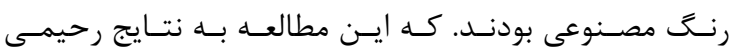

امروزه استفاده از رنگهاى خوراكى جه به صورت طبيعى و يا مصنوعى جهت خوش رنت و خوشمزه كردن غذا بسـيار رواج

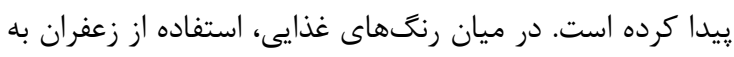

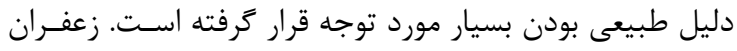

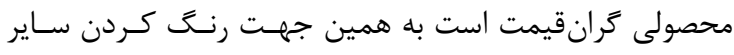

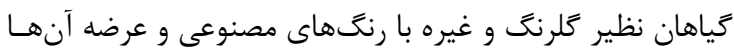

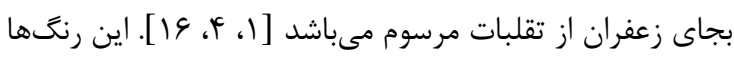

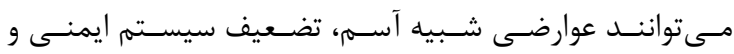


محصولات قنادى شهر قم انجام دادند رنغ كينولين شايعترين

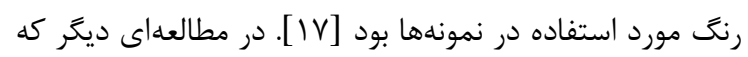

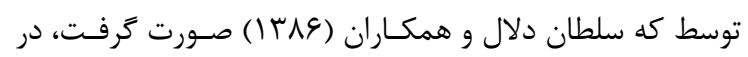

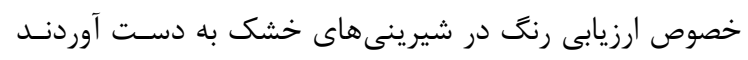

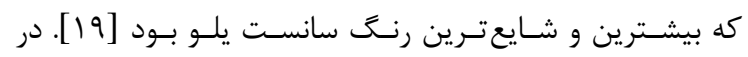

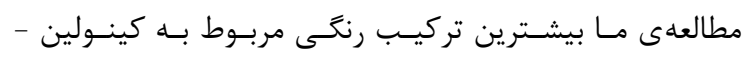

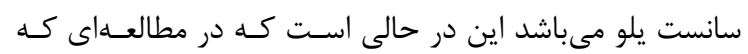

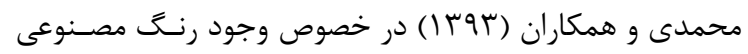

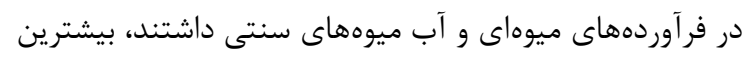

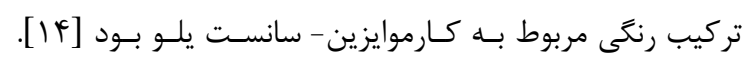

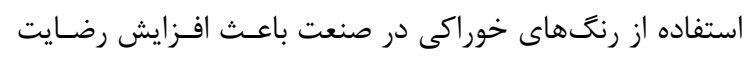

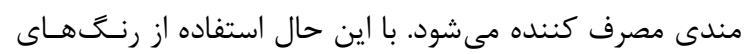

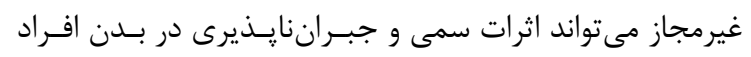

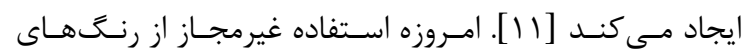

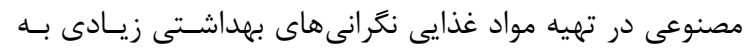

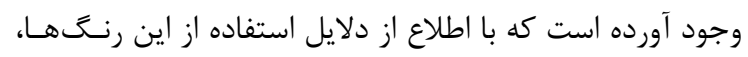

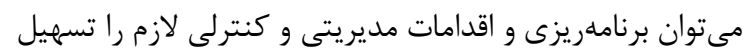

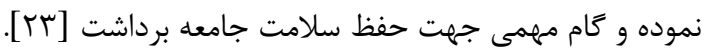

\section{نتيجه تيرى}

زعفران و محصولات زعفرانى امروزه از محبوبيت زيـادى در

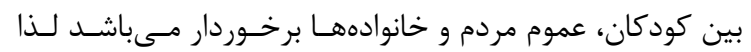

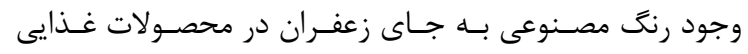

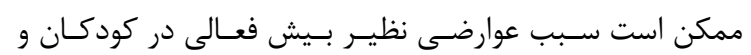

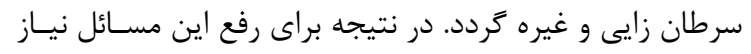

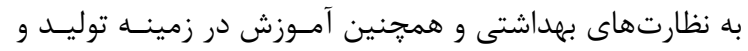

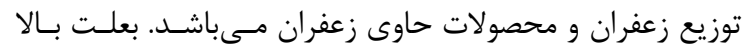

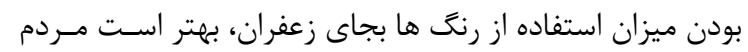

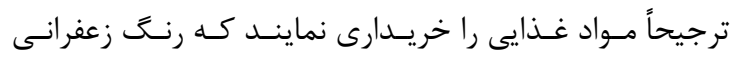
نداشته باشند.

\section{تشكر و قدردانى}

از معاونت محترم تحقيقات و فناورى دانشعاه علوم يزشـكى ولى

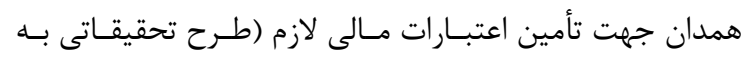

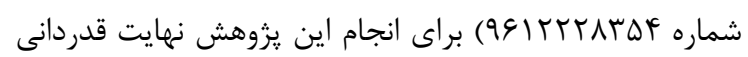
را دارد. - ماره

تضاد منافع اين مطالعه براى نويسندكًان هيجَّّنه تضاد منافعى نداشته

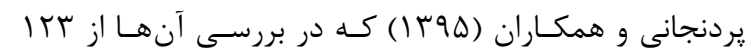

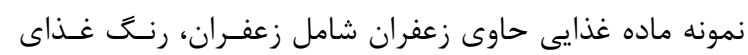

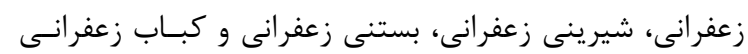

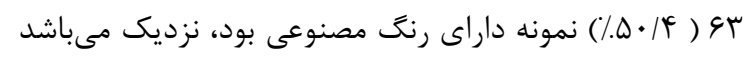

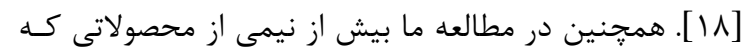

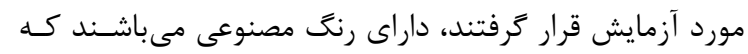

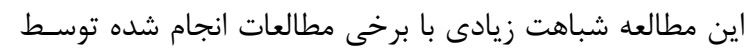

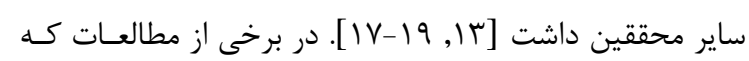

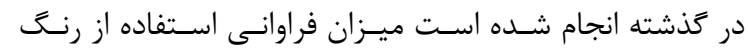

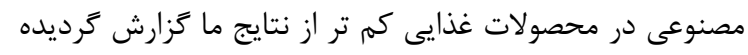

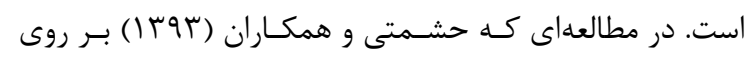
رنح بعا نمونه بستنى سنتى در شهرستان كرج انجـام دادنـد

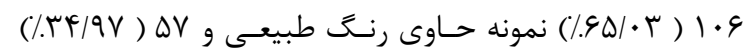

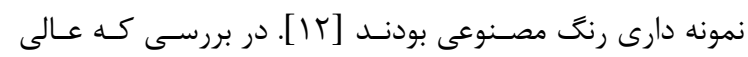

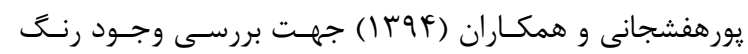

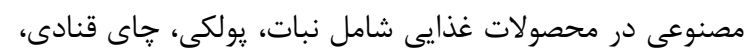

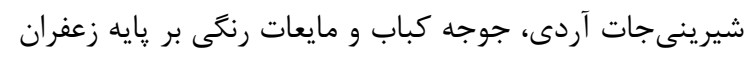

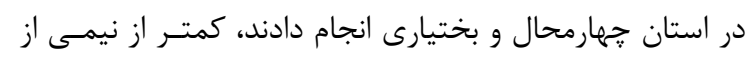

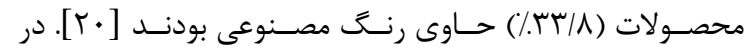

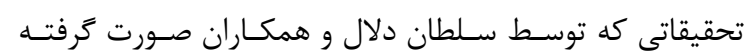

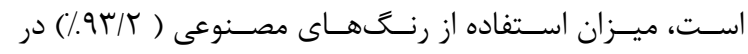

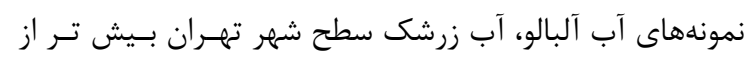

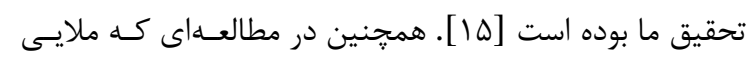

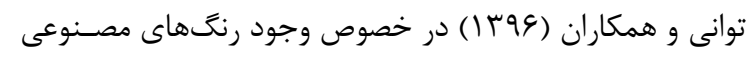

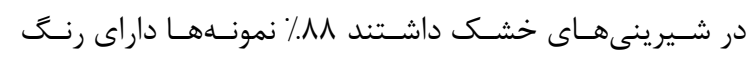

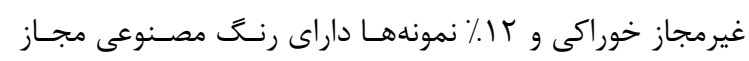

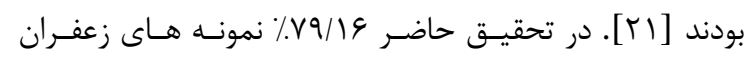

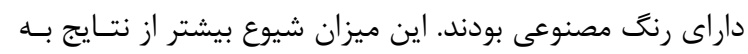

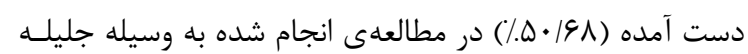

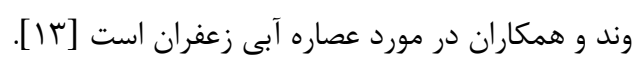

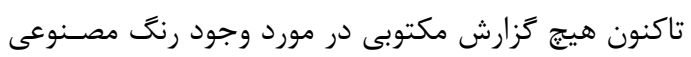

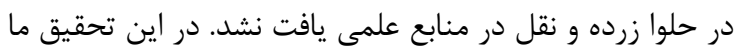

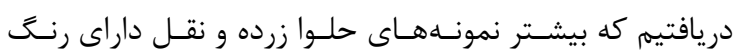

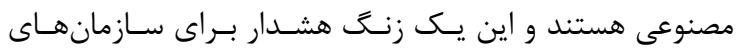
نظارتى مىباشد. در مطالعهى حاضر رنگ مصنوعى در نبات (هآ//) در تعداد

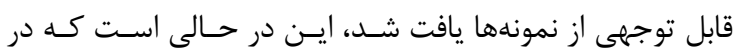

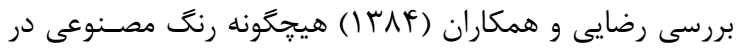

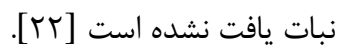
در تحقيق حاضر شايعترين رنگ مورد استفاده در نمونههـا

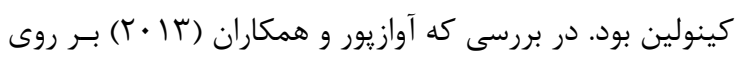

Food additives and hyperactive behaviour in 3-yearold and 8/9-year-old children in the community' by

The Department of Child Health.The lancet. 2007; 
370(9598):1524-1525. DOI: $\quad$ 10.1016/S0140-6736(07) 61306-3

2. Abdullaev F. Cancer chemopreventive and tumoricidal properties of saffro(Crocus sativus L.). Experimental Biology and Medicine. 2002; 227(1): 20-25.

3. Hosseinzadeh $\mathrm{H}$, Younesi $\mathrm{H}$. Petal and stigma extracts of Crocus sativus L. have antinociceptive and antiinflammatory effects in mice. BMC Pharmacology. 2002; 2: 1-8. DOI: 10.1186/1471-2210-2-7

4. Zarghami N S, Heinz D E. The volatile constituents of saffron, Lebensmittel-Wissenschaft and Technologie Food Science and Technology. 1971;4:41-45

5. Hagh-Nazari S, Keifi N. Saffron and various fraud manners in its production and trades. In II International Symposium on Saffron Biology and Technology. 2006; 739: 411-416.

6. ISIRI. Institute of Standards and Industrial Research of Iran, Ice cream - Specifications and test methods. ISIRI No. 2450. Fith erevision, 2008. (Persion)

7. ISIRI. Institute of Standards and Industrial Research of Iran, Tar additive color (test method):ISIRI No. 2634, first revision, 1988. (Persion)

8. Loskutov A, V., Beninger C, W., Hosfield G, L,.Sink K, C. Development of an improved procedure for extraction and quantification of safranal in stigmas of Crocus sativus L. using high performance liquid chromatography, Food Chemistry. 2000, 69(2): 87-95. DOI: https://doi.org/10.1016/S0308-8146(99)00246-0

9. Revision C, Tomes F, Collins C, Robert L, Sprado M, Shackelford D, et al. Proposed testing guidelines for developmental toxicity studies. J Regulatory Toxicol and Pharmacol. 2002;30:39-44.

10. Nigg JT, Lewis K, Edinger T, Falk M. Meta-analysis of attention-deficit/hyperactivity disorder or attentiondeficit/hyperactivity disorder symptoms, restriction diet, and synthetic food color additives. Journal of the American Academy of Child \& Adolescent Psychiatry. 2012 1;51 (1):86-97. DOI: 10.1016/j.jaac.2011.10.015

11. Arast Y, Mohammadian M, Noruzi M, Ramoz Z. Evaluating the trend of oral intakes in confectionary products in Qom. Department of Toxicology, School of Health Qom University of Medical Sciences Qom, Iran. 2013;15(3):62-64. (Persion)

12. Heshmati A, Hakhim s, Safari A. Kh, Afshar A, Amini Kh, Rabi N, Nasiri E. Existence and type of artificial color in traditional nuts and ice cream delivered in Karaj city. Scienitific and Research Journal Alborz University Of Medical Sciences. 2014;3(3):165-170. (Persion)

13. Jalilevand F, Rahiminiaraki A, Sadeghiniaraki A, HadizadeSaffari R. investigation of artificial colors in saffron aqueous extract of restaurants in Qazvin province in 2008. National conference on Environmental Health. 2009; 2666-2673. (Persion)

14. Mohammadi HR, Vahedi S, Hajimahmoodi M,
Nadjarian A, Salsali M, Shokrzadeh M. A Survey on the Use of Synthetic and Natural Fruit Colures in non-certified Juice and Fruit Products in Tehran, Iran.Journal Of Mazandaran Univ Med Sci. 2014; 24(120):159-172. (Persion)

15. Soltandalal M, Vahedi S, Najjarian A, Dastbaze A, Kaffashi T, Pirhadi E. The study of the frequency of unauthorized dye consumption in cherry juice and barberry juice supplied in Tehran. Payavardsalamat. 2008; 2(1-2): 55-62. (Persion)

16. Sereshti H, Poursorkh Z, Aliakbarzadeh G, Zarre S, Ataolahi S. An image analysis of TLC patterns for quality control of saffron based on soil salinity effect: A strategy for data (pre)-processing. Food Chemistry. 2018; 239: 831-839. DOI: https://doi.org/10.1016/j. foodchem.2017.07.012.

17. Avazpour M, seifipour F, abdi J, nabavi T, zamanianazodi M. Detection of dyes in confectionery products using thin-layer chromatography. Iranian Journal of Nutrition Sciences and Food Technology. 2013; 8(3): 73-78. (Persion)

18. Rahimi-pardanjani $\mathrm{S}$, kiani $\mathrm{M}$, ezzati $\mathrm{P}$, Pourmohammadi B, Biabani J, Torabi $\mathrm{H}$, et al. Determination of frequency of consumable colors in saffron foods in Yazd city using thin layer chromatography. Research Quarterly Journal Of University Medical Sciences Mashhad. 2016; 19 (61): 1-7. (Persion)

19. Soltandalal M, Mohamadi H-R, Dastbaz A, Mohamadi S, Salsali M, Arasteh M, et al. The state of consumable colors in dry sweets in southern Tehran using thin-layer chromatography. Journal of Gorgan university of Medical sciences. 2006; 9(9): 73-78. (Persion)

20. AlipourHafshejani F, MahdaviHafshejani F. Determine the prevalence of food contamination to synthetic colors with thin layer chromatography in Shahrekord. Shahrekord University of Medical Sciences 2016; 17(6): 103-112. (Persion)

21. Molaei Tavani S, Nobari S, Ghasemi R, Mazloomi S. Survey the Authorized and Unauthorized Food Colorings Consumption in the Food Supply of Nazarabad Township in 1395. Environmental Health Engineering. 2017; 4 (4): 299-306. (Persion)

22. Rezaei R, Mirlohi M, Maraccy MR, Vahid Dastjerdi M. Exposure Estimation to Tartrazine through Traditional Hard Candies (Nabat and Poulaki) in Iran, Isfahan Province. Health Research Journal. 2015; 11(3): 604-612. (Persion)

23. Khosravimashizi R, Yunesian M, Galoy E. Investigating the reasons for the unauthorized use of artificial colors in food preparation by hierarchical analysis method. Journal of Food Hygiene. 2016; 6(1): 75-86. 